\title{
Feasibility and effectiveness of expectant management of pneumothorax in newborns
}

\author{
D. Dobryanskyy ${ }^{1}$, A. Menshykova ${ }^{1,2}$, S. Vereshchak ${ }^{1}$, Z. Salabay ${ }^{2}$ \\ ${ }^{1}$ Department of Paediatrics \#2, Lviv National Medical University, Lviv, Ukraine \\ ${ }^{2}$ Neonatal Intensive Care Unit, Lviv Regional Clinical Hospital, Lviv, Ukraine
}

\section{Background and aims}

Pneumothorax is an important and common complication in newborns infants with lung diseases, especially if they need any respiratory support. This complication is associated with increased morbidity and mortality and does occur despite optimal exogenous surfactant and ventilation strategy use. The aim of this study was to evaluate the outcomes of expectant management of pneumothorax without chest-tube insertion.

\section{Methods}

Forty-five successively admitted newborns (8 terms and 37 preterms) with pneumothorax were included into a retrospective study. The prevalence of perinatal risk factors, morbidity, respiratory management data and mortality were compared in infants needed a chest-tube insertion versus those who were treated without this intervention.

\section{Results}

- Pneumothorax occurred in a median (IQR) age of 2 (1-2) days and in 22 cases (49\%) it was a tension one.

- Only $6(13 \%)$ infants (5 of them were preterms) needed chest tube insertion. All of them were on mechanical ventilation, had a tension pneumothorax and were initially treated with needle aspiration. In three cases (50\%) subsequent high frequency oscillatory ventilation (HFOV) was used.

- 39 infants who were cared without a chest tube were not different in terms of gestational age, birth weight and preceding ventilator settings. $16(41 \%)$ of them had a tension pneumothorax, 18 (46\%) were punctured and 21 (54\%) were managed expectantly. Initially they more commonly were on CPAP or NIV ( $54 \%$ vs. $0 \% ; p<0.05$ ), were intubated only after occurrence of pneumothorax (45\% vs. $0 \%$; $p<0.05$ ) but had lower incidence of severe RDS ( $21 \%$ vs. $50 \%$; p>0.05), severe IVH (18\% vs. $67 \%$; $p<0.05)$ and mortality (21\% vs. $100 \%$; $<<0.05)$.

\section{Comparative lung morbidity in the groups}

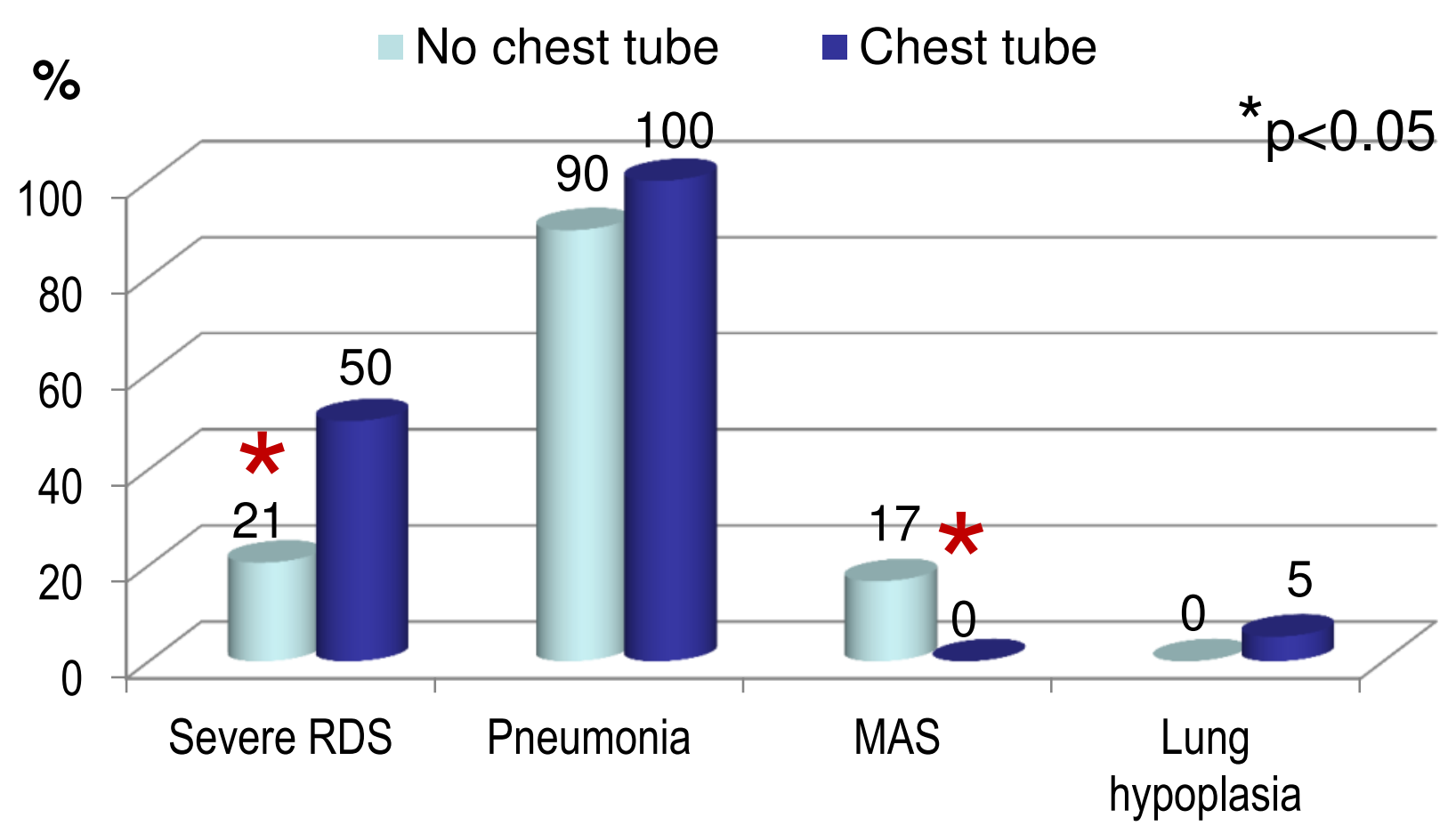

HFOV was successful in 19 (49\%) cases from this group including the all infants with a tension pneumothorax.

\begin{tabular}{|c|c|c|c|}
\hline Characteristics & $\begin{array}{c}\text { Chest tube } \\
(n=6)\end{array}$ & $\begin{array}{c}\text { No chest } \\
\text { tube }(n=39)\end{array}$ & $p$ \\
\hline Birth weight, gr. ${ }^{1}$ & $1781.67(1123.39)$ & $2054.1(845.23)$ & $>.05$ \\
\hline 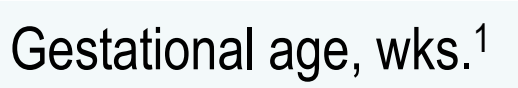 & $31,50(5,32)$ & $32,95(4,27)$ & $>.05$ \\
\hline Preterms ${ }^{2}$ & $5(83)$ & $32(82)$ & $>.05$ \\
\hline Surfactant ${ }^{2}$ & $4(67)$ & $11(28)$ & $>.05$ \\
\hline $\begin{array}{l}\text { Pneumothorax } \\
\text { occurrence age, days }{ }^{3}\end{array}$ & $2.0(1-6)$ & $1.0(0-4)$ & $>.05$ \\
\hline $\begin{array}{l}\text { Tension } \\
\text { pneumothorax² }\end{array}$ & $6(100)$ & $16(41)$ & $<.05$ \\
\hline $\begin{array}{l}\text { Two-sided } \\
\text { pneumothorax² }\end{array}$ & $2(33)$ & $8(21)$ & $>.05$ \\
\hline Severe IVH² & $4(67)$ & 7 (18) & $<.05$ \\
\hline Died $^{2}$ & $6(100)$ & $8(21)$ & $<.05$ \\
\hline
\end{tabular}

Notes. 1 - mean (SD); 2 - number of cases (\%); 3 - median (min-max).

\begin{tabular}{|c|c|c|c|}
\hline Parameter & $\begin{array}{l}\text { Chest tube } \\
\qquad(n=6)\end{array}$ & $\begin{array}{c}\text { No chest } \\
\text { tube }(n=39)\end{array}$ & $\mathbf{p}$ \\
\hline $\mathrm{CPAP}^{1}$ & $0(0)$ & $19(49)$ & $<.05$ \\
\hline Non-invasive ventilation ${ }^{1}$ & $0(0)$ & $2(5)$ & $>.05$ \\
\hline Endotracheal SIMV1 & $6(100)$ & $16(41)$ & $<.01$ \\
\hline $\mathrm{FiO}_{2}, \% 2$ & $46.67(27.14)$ & $46.97(16.98)$ & $>.05$ \\
\hline $\mathrm{PIP}, \mathrm{cm} \mathrm{H}_{2} \mathrm{O}^{2}$ & $20.5(4.93)$ & $20.75(3.64)$ & $>.05$ \\
\hline PEEP, $\mathrm{cm} \mathrm{H}_{2} \mathrm{O}^{2}$ & $5.0(0)$ & $5.56(0.79)$ & $>.05$ \\
\hline Ventilation frequency $(/ \mathrm{min})^{2}$ & $39.17(11.14)$ & $44.88(14.3)$ & $>.05$ \\
\hline
\end{tabular}

Notes. 1 - number of cases (\%); 2 - mean (SD).

\section{Specific treatment methods in the groups}

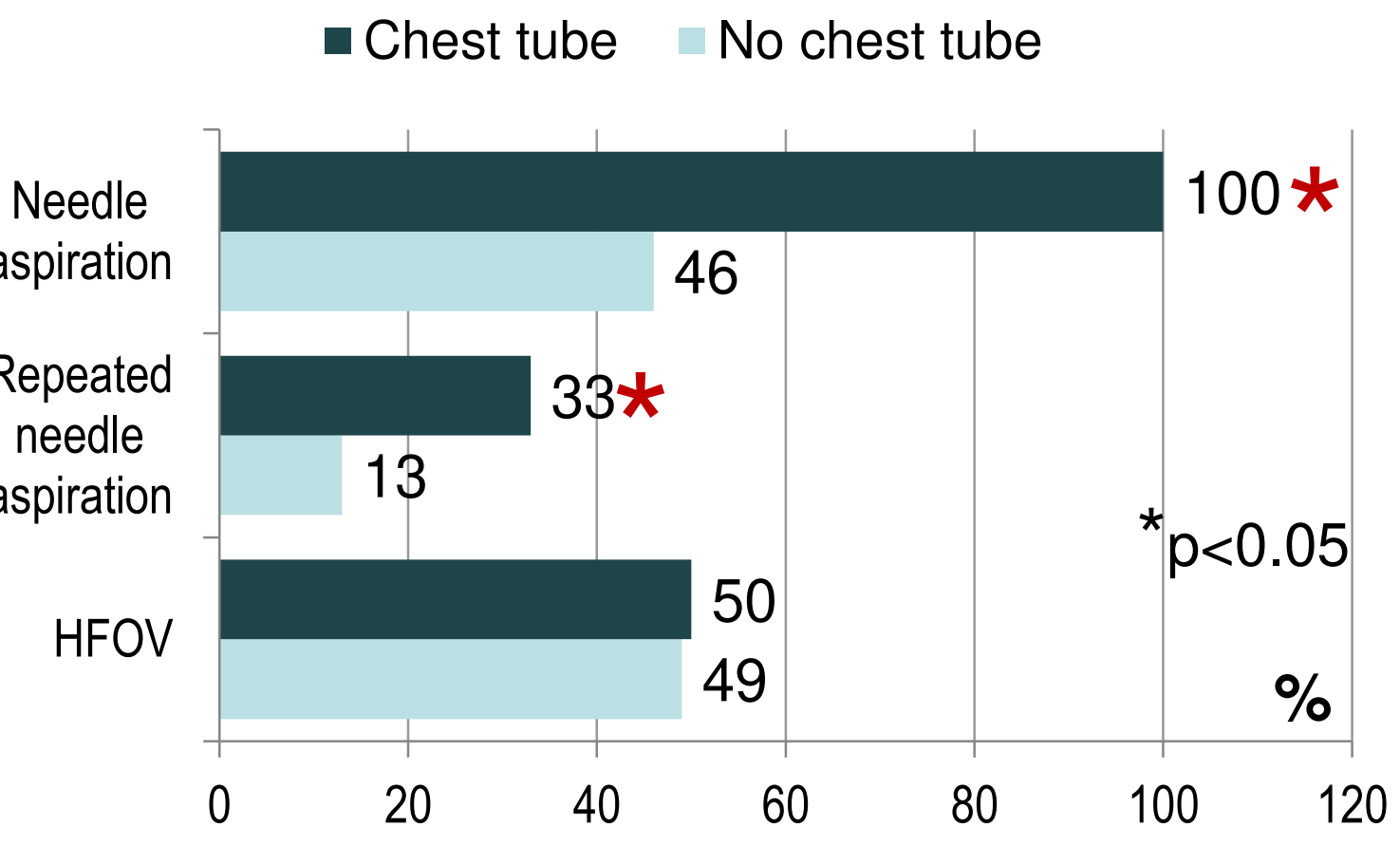

\section{Conclusions}

Majority of term and preterm newborns with even a tension pneumothorax could be successfully cared without chest-tube placement. Need for chest-tube insertion worsens prognosis. 